\title{
Galerkin multi-wavelet bases and mesh points method to solve integral equation with singular logarithmic kernel
}

\author{
Mohsen Rabbani ${ }^{1} \cdot$ Amir Ali Tabatabai-adnani $^{2} \cdot$ Mandana Tamizkar $^{1}$
}

Received: 19 November 2017 / Accepted: 9 January 2018/ Published online: 25 January 2018

(C) The Author(s) 2018. This article is an open access publication

\begin{abstract}
In this article, we use an effective coupled technique to solve singular integral equations with logarithmic kernel which it occurs in different branches of sciences such as mathematical physics, mechanics, isotropic elastic, etc. At first, we approximate the singular kernel by choosing suitable mesh points and multi-wavelet bases then, Galerkin method is used for solving integral equations. In addition, for simplification and to achieve to a more accuracy, we convert integral equation with logarithmic kernel to integro-differential equation with weakly singular kernel which is solvable with the proposed method. The numerical results show the high accuracy of the solution.
\end{abstract}

Keywords Integral equation · Integro-differential equation · Singular kernel · Wavelet bases · Legendre polynomial · Orthonormal

Mathematics Subject Classification $42 \mathrm{~B} 20 \cdot 47 \mathrm{Gxx} \cdot 42 \mathrm{C} 40 \cdot 42 \mathrm{C} 10$

\section{Introduction}

A singular integral equation occurs in some concepts of engineering mechanics, such as elasticity, plasticity, and aerodynamics (see [7, 12]). Cauchy integral equation is a kind of singular integral equation that introduced in $[8,9]$, such that for solving this problem, an approximate method by polynomials and collocation points is given by [5]. Abdou and Naser [1] applied orthogonal polynomials to solve Cauchy singular integral equations. In addition, the above problem in [6] is solved with the help of Gaussian quadrature and collocation method. Srivastav and Zhang [18] used general quadrature-collocation nodes to solve Cauchy singular integral equation.

Mohsen Rabbani

mrabbani@iausari.ac.ir

Amir Ali Tabatabai-adnani

a.t.adnani@gmail.com

Mandana Tamizkar

tamizkar.m@gmail.com

1 Department of Mathematics, Sari Branch, Islamic Azad University, Sari, Iran

2 Department of Mathematics, Central Branch, Islamic Azad University, Tehran, Iran
Some applications of integro-differential equations can be seen in biological issues, nanotechnology and signal processing, etc. For extra details, see [3, 19, 20]. Because analytical solutions of integro-differential equations are difficult, therefore, numerical methods can be effectual such as hybrid Euler-Taylor matrix method, the integral mean value theorem, non-standard finite difference, and multi-wavelet Petrov-Galerkin method in $[4,10,11,13]$, respectively.

Thus, singular integral equation has a particular importance in different branches of science especially integral equation with logarithmic kernel.

Consider Fredholm integral equation with logarithmic kernel:

$u(x)-\int_{0}^{1} \ln |x-y|^{\alpha} u(y) \mathrm{d} y=f(x) \quad 0 \leq x \leq 1$

where $f \in L^{2}[0,1]$ is known function and $u$ is unknown function to be determined. By differentiating both sides of Eq. (1) with respect to $x$, we obtain singular integro-differential equation as follows:

$$
u^{\prime}(x)-\alpha \int_{0}^{1} \frac{u(y)}{(x-y)} \mathrm{d} y=f^{\prime}(x) \quad 0 \leq x \leq 1 .
$$

Several methods have been proposed for solving the integral equation with logarithmic kernel. BPFs and Taylor 
series expansion method are introduced in [16]; wavelet method is presented in [17]. At first, we approximate logarithmic kernel using suitable mesh points and multiwavelet basis, and then, we use Galerkin method to solve (1). In addition, on the other hand, as a second method instead of solving integral equation with logarithmic kernel, we solve integro-differential equation with singular kernel (2), so we approximate singular kernel similar to the above estimate. In this regard, we use orthonormal bases which is produced by Legendre polynomials to obtain the solution of integro-differential equation. Because the elements of the bases are orthonormal, so the number of operations reduces. In the following, we introduce multiwavelet bases [2].

\section{Multi-wavelet}

We use multi-wavelet as a basis of $L^{2}[0,1]$ see [2], where $[0,1]$ is divided to $2^{m}$ subintervals and subspaces $s_{m}^{k}$ are defined by polynomials with degree less than $k$ :

$s_{m}^{k}=$

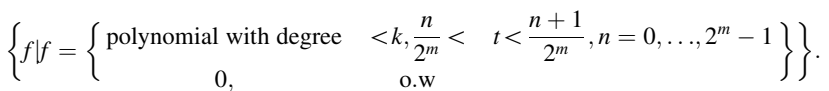

It is obvious that $\operatorname{dim}\left(S_{m}^{k}\right)=2^{m} k$ and $S_{0}^{k} \subset S_{1}^{k} \subset \cdots$. Assume that $R_{m}^{k}$ is the complement of $S_{m}^{k}$ in $S_{m+1}^{k}$, then

$S_{m+1}^{k}=S_{m}^{k} \oplus R_{m}^{k}, \quad S_{m}^{k} \perp R_{m}^{k}$,

also

$S_{m}^{k}=S_{m-1}^{k} \oplus R_{m-1}^{k}=S_{0}^{k} \oplus_{p=0}^{m-1} R_{p}^{k}$,

and with assumption $S^{k}=\cup_{m=o}^{\infty} S_{m}^{k}$, then $S^{k}=L^{2}[0,1]$ [2]. Some applications of the multi-wavelet bases can be seen in $[13,14]$. Such that in $[13,14]$, the above basis used for trial and test spaces in Petrov-Galerkin method to solve integro-differential system and integral equation, respectively. In addition, in [15] multi-wavelet bases is used to modify the integrand function approximation coefficient to solve Hammerstein integral equations.

\section{Kernel approximation}

Assuming that the solution of integral equation is in the space $L^{2}[0,1]$, we divide horizontal axis on the interval $[0,1]$ to points $x_{0}, x_{1}, \ldots, x_{m}$ and also the vertical axis on the interval $[0,1]$ to points $y_{0}, y_{1}, \ldots, y_{m}$ for mesh points selection.

On every one of square as $I_{i j}=\left[x_{i-1}, x_{i}\right] \times\left[y_{j-1}, y_{j}\right]$, we approximate the kernel using orthonormal multi-wavelet bases. We consider to $X_{n} \times X_{n}$ as a subspace of $L^{2}[0,1] \times$ $[0,1]$ where $X_{n}$ introduced in [14], then the kernel $k(x, y)$ in space $X_{n} \times X_{n}$ is approximated as follows:

$k(x, y)=\sum_{i=0}^{n} \sum_{j=0}^{n} k_{i j} u_{i}(x) u_{j}(y)$.

Orthonormal basis $u_{i}$ can be selected from Legendre orthonormal polynomials or orthonormal Legendre multiwavelet bases [13]. Therefore, the coefficients of the kernel approximation can be calculated to this form:

$$
\begin{aligned}
k_{i j} & =\int_{0}^{1} \int_{0}^{1} k(x, y) u_{i}(x) u_{j}(y) \mathrm{d} x \mathrm{~d} y \\
& =\int_{0}^{1} \int_{0}^{1} \widehat{k}_{i j}(x, y) \mathrm{d} x \mathrm{~d} y .
\end{aligned}
$$

To compute the above integral, we calculate the integral by assuming $y_{i}=x_{i}$ for $i=0,1, \ldots, m$ and integration on the squares upper and lower of the line $y=x$ and on the squares that the line $y=x$ passes on those diagonals, so in the following form:

$$
\begin{aligned}
k_{i j} & =\int_{0}^{1} \int_{0}^{1} \widehat{k}_{i j}(x, y) \mathrm{d} x \mathrm{~d} y \\
& =\sum_{p, q=1}^{m} \iint_{I_{p q}} \widehat{k}_{i j}(x, y) \mathrm{d} x \mathrm{~d} y \\
& p \neq q \\
& +\sum_{p=1}^{m} \iint_{I_{p p}} \widehat{k}_{i j}(x, y) \mathrm{d} x \mathrm{~d} y,
\end{aligned}
$$

for computing the first integral right side of (5), we select central point of the square $I_{p q}, p \neq q$, as follows:

$$
\begin{aligned}
\left.\iint_{\substack{I_{p q p} \neq q\\
}} \widehat{k}_{i j}(x, y) \mathrm{d} x \mathrm{~d} y \simeq \text { (the area of the square } I_{p q}\right) \widehat{k}_{i j}\left(x_{M}, y_{M}\right) \\
\quad=\left(x_{p}-x_{p-1}\right)\left(y_{q}-y_{q-1}\right) \widehat{k}_{i j}\left(x_{p}-\frac{h}{2}, y_{q}-\frac{h}{2}\right),
\end{aligned}
$$

by the equidistant points $x_{0}=y_{0}=0, x_{p}=y_{p}$ for all $p=$ $1,2, \ldots, n$ we have

$=h^{2} \widehat{k}_{i j}\left(\frac{(2 p-1) h}{2}, \frac{(2 q-1) h}{2}\right)$.

To compute second integral in the right side of (5), we focus on the squares located on the line $y=x$ which the squares $I_{p q}$ are divided to lower and upper triangles both sides of the line $y=x$ and multiply the area of the triangle to $\widehat{k}_{i j}(x, y)$ at the central point of triangle. For this purpose, we suppose the coordinates of the points $A, B, C, D$ as follows: 


$$
\begin{aligned}
& A=\left(x_{p-1}, y_{p-1}\right), B=\left(x_{p}, y_{p-1}\right), C=\left(x_{p}, y_{p}\right), \\
& D=\left(x_{p-1}, y_{p}\right),
\end{aligned}
$$

Therefore, central points are as:

$$
\begin{aligned}
G_{1} & =\left(\frac{(3 p-1) h}{3}, \frac{(3 p-2) h}{3}\right), \\
G_{2} & =\left(\frac{(3 p-2) h}{3}, \frac{(3 p-1) h}{3}\right),
\end{aligned}
$$

also we can write

$$
\begin{aligned}
\iint_{I_{p p}} \widehat{k}_{i j}(x, y) \mathrm{d} x \mathrm{~d} y= & \left(S_{A B C}\right) \widehat{k}_{i j}\left(G_{1}\right)+\left(S_{A D C}\right) \widehat{k}_{i j}\left(G_{2}\right) \\
= & \frac{h^{2}}{2}\left[\widehat{k}_{i j}\left(\frac{(3 p-1) h}{3}, \frac{(3 p-2) h}{3}\right)\right. \\
& \left.+\widehat{k}_{i j}\left(\frac{(3 p-2) h}{3}, \frac{(3 p-1) h}{3}\right)\right] .
\end{aligned}
$$

According to $(5,6,7)$, the coefficients $k_{i j}$ for kernel approximation with $h=\frac{1}{m^{2}}$ and sufficiently large $m$ can be calculated upper and lower of the line $y=x$ in the case of $p \neq q$ and on the line $y=x$ in the case of $p=q$ as follows:

$$
\begin{aligned}
k_{i j} & =\frac{1}{m^{2}}\left\{\sum_{p=1}^{m-1} \sum_{q=p+1}^{m} k\left(\frac{(2 p-1)}{2 m}, \frac{(2 q-1)}{2 m}\right)\right. \\
& u_{i}\left(\frac{2 p-1}{2 m}\right) u_{j}\left(\frac{2 q-1}{2 m}\right)+\sum_{q=1}^{m-1} \sum_{p=q+1}^{m} k\left(\frac{(2 p-1)}{2 m}, \frac{(2 q-1)}{2 m}\right) \\
& \left.u_{i}\left(\frac{2 p-1}{2 m}\right) u_{j}\left(\frac{2 q-1}{2 m}\right)\right\}+\frac{1}{2 m^{2}} \sum_{p=1}^{m}\left\{k\left(\frac{(3 p-1)}{3 m}, \frac{(3 p-2)}{3 m}\right)\right. \\
& u_{i}\left(\frac{(3 p-1)}{3 m}\right) u_{j}\left(\frac{(3 p-2)}{3 m}\right)+k\left(\frac{(3 p-2)}{3 m}, \frac{(3 p-1)}{3 m}\right) \\
& \left.u_{i}\left(\frac{(3 p-2)}{3 m}\right) u_{j}\left(\frac{(3 p-1)}{3 m}\right)\right\} .
\end{aligned}
$$

By the formula (8), we can solve singularity problem for some kernels, $k(x, y)=\log |x-y|^{\alpha}$ and $k(x, y)=\frac{1}{(x-y)^{\alpha}}$.

\section{Error analysis}

We consider $k(x, y)$ on every of $I_{p q} \subset[0,1] \times[0,1]$ subject to $p \neq q(p, q=1, \ldots, m)$, so there is not any singularity for $k(x, y)$, then $k(x, y) \in C\left[x_{p-1}, x_{p}\right] \times\left[y_{p-1}, y_{p}\right]$, where $p, q$ is given the above.

Lemma 1 Let $k(x, y) \in C\left(I_{p q}\right)=C\left[x_{p-1}, x_{p}\right] \times$ $\left[y_{q-1}, y_{q}\right] \subseteq L^{2}\left(I_{p q}\right)$, then

$$
\lim _{n \rightarrow \infty}\left\|k(x, y)-\sum_{i, j=0}^{n} k_{i j} u_{i}(x) u_{j}(y)\right\|_{2} \longrightarrow 0,
$$

where $k_{i, j} i, j=1,2, \ldots, n$ is given by (4) and $\left\{u_{i}\right\}_{i=1}^{n} \subseteq$ $S_{m}^{k}$ is as an orthonormal Legendre multi-wavelet bases.

\section{Proof}

$$
\begin{aligned}
\|r\|_{2}^{2}= & \left\|k(x, y)-\sum_{i, j=0}^{n} k_{i j} u_{i}(x) u_{j}(y)\right\|_{2}^{2} \\
= & \left\langle k(x, y)-\sum_{i, j=0}^{n} k_{i j} u_{i}(x) u_{j}(y), k(x, y)-\sum_{i, j=0}^{n} k_{i j} u_{i}(x) u_{j}(y)\right\rangle \\
= & \|k(x, y)\|_{2}^{2}-2 \sum_{i, j=0}^{n}\left\langle k(x, y), u_{i}(x) u_{j}(y)\right\rangle^{2} \\
& +\sum_{i, j=0}^{n}\left[k_{i j}-\left\langle k(x, y), u_{i}(x) u_{j}(y)\right\rangle\right]^{2} .
\end{aligned}
$$

Since $\left\{u_{i}(x), u_{j}(y)\right\}_{i, j=1}^{n}$ is as an orthonormal multi-wavelet basis, thus, last expression of (9) is equal to zero, so $\|r\|_{2}$ is minimized. In the other hand according to the "Weiers tras's approximation theorem", there exists a sequence of polynomials such as $Q_{n, n}(x, y)$, subject to

$\forall \varepsilon>0, \quad \max \quad\left|k(x, y)-Q_{n, n}(x, y)\right|<\varepsilon$.

$$
\begin{gathered}
(x, y) \in I_{p q} \\
p, q=1 \\
p \neq q
\end{gathered}
$$

Therefore, because $r(x, y)$ is minimized for each $(x, y) \in I_{p q}, p \neq q$, then

$$
\begin{aligned}
\|r\|_{2}^{2} & =\left\|k(x, y)-\sum_{i, j=0}^{n} k_{i j} u_{i}(x) u_{j}(y)\right\|_{2}^{2} \\
& \leq\left\|k(x, y)-Q_{n, n}(x, y)\right\|_{2}^{2} \\
& =\int_{0}^{1} \int_{0}^{1}\left|k(x, y)-Q_{n, n}(x, y)\right|^{2} \mathrm{~d} x \mathrm{~d} y \\
& \leq \int_{0}^{1} \int_{0}^{1} \varepsilon^{2} \mathrm{~d} x \mathrm{~d} y=\varepsilon^{2} .
\end{aligned}
$$

So, $\|r\|_{2} \leq \varepsilon$. Since $\varepsilon$ is arbitrary then $\lim _{n \rightarrow \infty}\|r\|_{2}=0$. Now, we consider error analysis for estimating $k(x, y)$ on every squares of $I_{p p}, p=1, \ldots, m$ on the line $y=x$.

Lemma 2 Let $\widehat{k}_{i j}(x, y)$ has two times continuous partial derivative in neighbourhood of $X_{0}=\left(x_{p-1}, y_{p-1}\right)$ in $I_{p p}=$ $\left[x_{p-1}, x_{p}\right] \times\left[y_{p-1}, y_{p}\right] \subset[0,1] \times[0,1], x_{p}=y_{p}=p h, p=1$, $\ldots, n$, then error expression is proportional with $O\left(h^{4}\right)$ : 


$$
\begin{array}{r}
E=\int_{y_{p-1}}^{y_{p}} \int_{x_{p-1}}^{x_{p}} \widehat{k}_{i j}(x, y) \mathrm{d} x \mathrm{~d} y-\frac{h^{2}}{2}\left[\widehat { k } _ { i j } \left(x_{p-1}+\frac{2 h}{3},\right.\right. \\
\left.\left.y_{p-1}+\frac{h}{3}\right)+\widehat{k}_{i j}\left(x_{p-1}+\frac{h}{3}, y_{p-1}+\frac{2 h}{3}\right)\right] .
\end{array}
$$

Proof We use Taylor expansion for $\widehat{k}_{i j}(x, y)$ in the case of two dimensional:

$$
\begin{aligned}
\widehat{k}_{i j}(x, y)= & \widehat{k}_{i j}\left(x_{p-1}, y_{p-1}\right) \\
& +\left.\sum_{i=1}^{n} \frac{1}{i !}\left[\left(x-x_{p-1}\right) \frac{\partial}{\partial x}+\left(y-y_{p-1}\right) \frac{\partial}{\partial y}\right]^{i} \widehat{k}_{i j}(x, y)\right|_{\substack{x=x_{p-1} \\
y=y_{p-1}}} .
\end{aligned}
$$

Thus, for second expression of (10), we have

$$
\begin{aligned}
& \frac{h^{2}}{2}\left[\widehat{k}_{i j}\left(x_{p-1}+\frac{2 h}{3}, y_{p-1}+\frac{h}{3}\right)\right. \\
& \left.+\widehat{k}_{i j}\left(x_{p-1}+\frac{h}{3}, y_{p-1}+\frac{2 h}{3}\right)\right] \\
& =h^{2} \widehat{k}_{i j}\left(x_{p-1}, y_{p-1}\right)+\left(\frac{h^{3}}{2} \frac{\partial}{\partial x} \widehat{k}_{i j}(x, y)+\frac{h^{3}}{2} \frac{\partial}{\partial y} \widehat{k}_{i j}(x, y)\right. \\
& +\frac{5 h^{4}}{36} \frac{\partial^{2}}{\partial x^{2}} \widehat{k}_{i j}(x, y)+\frac{5 h^{4}}{36} \frac{\partial^{2} \widehat{k}_{i j}}{\partial y^{2}}(x, y)+\frac{2 h^{4}}{9} \frac{\partial^{2} \widehat{k}_{i j}}{\partial x \partial y}(x, y) \\
& +\ldots) \mid \begin{array}{l}
x=x_{p-1} \\
y=y_{p-1}
\end{array} \text {. }
\end{aligned}
$$

By substituting (11-12) in (10), we obtain expression of error:

$$
\begin{aligned}
E= & \frac{h^{4}}{36}\left(\widehat{k}_{i j x x}\left(x_{p-1}, y_{p-1}\right)+\widehat{k}_{i j y y}\left(x_{p-1}, y_{p-1}\right)\right. \\
& \left.+\widehat{k}_{i j x y}\left(x_{p-1}, y_{p-1}\right)\right)+\ldots
\end{aligned}
$$

where $\widehat{k}_{i j x x}\left(x_{p-1}, y_{p-1}\right)=\left.\frac{\partial^{2}}{\partial x^{2}} \widehat{k}_{i j}(x, y)\right|_{\substack{x=x_{p-1} \\ y=y_{p-1}}} ^{x}$ and we derive that $E \approx O\left(h^{4}\right)$. The formula (10) is suitable for integrals with singular points, such as $\left(x_{p-1}, y_{p-1}\right)$ is singularity points for $\hat{k}(x, y)$ in (4).

\section{Galerkin method}

After approximating of the kernel by (8) in Sect. 3, we solve singular integral equation (1) with Galerkin method in $S_{m}^{k} \subseteq L^{2}[0,1]$ spaces. To this end we set, $u(x)=\sum_{i=0}^{n} \alpha_{i} u_{i}(x)$; subject to $\left\{u_{i}\right\}_{i=0}^{n}$ is an orthonormal Legendre multi-wavelet basis of $S_{m}^{k}$ and $n=\operatorname{dim}\left(S_{m}^{k}\right)=2^{m} k$. By substituting the above formula and (8) in (1), we have

$$
\begin{aligned}
& \sum_{i=0}^{n} \alpha_{i} u_{i}(x)-\sum_{i=0}^{n} \sum_{j=0}^{n} k_{i j} u_{i}(x) \int_{0}^{1} u_{j}(y) \sum_{d=0}^{n} \alpha_{d} u_{d}(y) \mathrm{d} y \\
& \quad=f(x),
\end{aligned}
$$

since $u_{i}^{\prime} s$ are orthonormal, thus

$\sum_{i=0}^{n} \alpha_{i} u_{i}(x)-\sum_{i, j=0}^{n} k_{i j} \alpha_{j} u_{i}(x)=f(x)$

applying inner product on (13) we obtain

$$
\begin{aligned}
& \sum_{i=0}^{n} \alpha_{i}\left\langle u_{i}(x), u_{p}(x)\right\rangle-\sum_{i, j=0}^{n} k_{i j} \alpha_{j}\left\langle u_{i}(x), u_{p}(x)\right\rangle \\
& \quad=\left\langle f(x), u_{p}(x)\right\rangle .
\end{aligned}
$$

Therefore

$$
\alpha_{p}-\sum_{j=0}^{n} k_{p j} \alpha_{j}=f_{p} \quad p=0,1, \ldots, n .
$$

The matrix form of system (14) is $(I-K) \alpha=F$, where $I=\quad$ identity matrix, $\quad F=\left(f_{0}, f_{1}, \ldots, f_{n}\right)^{T}, \alpha=$ $\left(\alpha_{0}, \alpha_{1}, \ldots, \alpha_{n}\right)^{T}, k=\left[k_{i j}\right]_{i, j=0}^{n}, k_{i j}$ are coefficients of kernel approximation that is given by (8) and $f_{p}=\int_{0}^{1} f(x) u_{p}(x) \mathrm{d} x$.

\section{Numerical results}

We solve some problems to indicate ability of the proposed method. Numerical results are obtained by the mathematica10 software.

\section{Example. 1}

Consider Fredholm integral equations with logarithmic kernel:

$$
\begin{aligned}
& u(x)-\int_{0}^{1} \ln |x-y|^{\alpha} u(y) \mathrm{d} y \\
& =\alpha\left(\frac{x^{3}}{3} \ln \left(\frac{1-x}{x}\right)-\frac{1}{3} \ln (1-x)+\frac{x}{6}+\frac{1}{9}\right)+\left(1+\frac{\alpha}{3}\right) x^{2}, \\
& \text { exactsolution : } \mathrm{u}(\mathrm{x})=\mathrm{x}^{2} \text {. }
\end{aligned}
$$

To solve the above problem, we apply system of (14) and coefficients of kernel approximation (8). For $\alpha=1$ and 0.5 , the numerical results have a good accuracy in Tables 1 and 2 , respectively. 
Table 1 Computational results for Example. $1(\alpha=1)$

\begin{tabular}{lll}
\hline & $X_{n}=S_{0}^{6}, m=500$ & $X_{n}=S_{1}^{3}, m=500$ \\
\hline 0 & $3.5 \times 10^{-5}$ & $1.7 \times 10^{-5}$ \\
0.1 & $3.1 \times 10^{-5}$ & $3.6 \times 10^{-5}$ \\
0.2 & $2.9 \times 10^{-5}$ & $3.3 \times 10^{-5}$ \\
0.3 & $8.9 \times 10^{-6}$ & $7.8 \times 10^{-6}$ \\
0.4 & $3.5 \times 10^{-5}$ & $3.9 \times 10^{-5}$ \\
0.5 & $1.1 \times 10^{-4}$ & $1.1 \times 10^{-5}$ \\
0.6 & $1.8 \times 10^{-4}$ & $1.7 \times 10^{-4}$ \\
0.7 & $2.8 \times 10^{-4}$ & $2.7 \times 10^{-4}$ \\
0.8 & $4.2 \times 10^{-4}$ & $4.3 \times 10^{-4}$ \\
0.9 & $6.3 \times 10^{-4}$ & $6.5 \times 10^{-4}$ \\
1 & $9.7 \times 10^{-4}$ & $9.2 \times 10^{-4}$ \\
\hline
\end{tabular}

Table 2 Computational results for Example. $1(\alpha=0.5)$

\begin{tabular}{lll}
\hline & $X_{n}=S_{0}^{6}, m=500$ & $X_{n}=S_{1}^{3}, m=500$ \\
\hline 0 & $1.7 \times 10^{-5}$ & $9.2 \times 10^{-6}$ \\
0.1 & $1.4 \times 10^{-5}$ & $1.6 \times 10^{-5}$ \\
0.2 & $8.7 \times 10^{-6}$ & $1.0 \times 10^{-5}$ \\
0.3 & $9.0 \times 10^{-6}$ & $9.1 \times 10^{-6}$ \\
0.4 & $4.0 \times 10^{-5}$ & $4.2 \times 10^{-5}$ \\
0.5 & $8.5 \times 10^{-5}$ & $9.0 \times 10^{-5}$ \\
0.6 & $1.4 \times 10^{-4}$ & $1.3 \times 10^{-4}$ \\
0.7 & $2.0 \times 10^{-4}$ & $2.0 \times 10^{-4}$ \\
0.8 & $2.9 \times 10^{-4}$ & $2.9 \times 10^{-4}$ \\
0.9 & $4.0 \times 10^{-4}$ & $4.1 \times 10^{-4}$ \\
1 & $5.7 \times 10^{-4}$ & $5.6 \times 10^{-4}$ \\
\hline
\end{tabular}

\section{Example. 2}

As mentioned in (2), Fredholm integral equations with logarithmic kernel in Example. 1 can be converted to a singular integro-differential equations:

$$
\begin{aligned}
& u^{\prime}(x)-\alpha \int_{0}^{1} \frac{u(y)}{(x-y)} \mathrm{d} y \\
& \quad=\alpha\left(x^{2} \ln \left(\frac{1-x}{x}\right)+\frac{1-x^{2}}{3(1-x)}+\frac{1}{6}\right)+2\left(1+\frac{\alpha}{3}\right) x, \\
& u(0)=0,
\end{aligned}
$$

exactsolution : $\mathrm{u}(\mathrm{x})=\mathrm{x}^{2}$.

Using system of (14) and coefficients of kernel approximation (8), we show absolute errors in some points in Tables 3 and 4.
Table 3 Computational results for Example. $2(\alpha=1)$

\begin{tabular}{lll}
\hline & $X_{n}=S_{0}^{6}, m=500$ & $X_{n}=S_{1}^{3}, m=500$ \\
\hline 0 & $1.6 \times 10^{-16}$ & $7.1 \times 10^{-17}$ \\
0.1 & $2.1 \times 10^{-7}$ & $1.4 \times 10^{-5}$ \\
0.2 & $5.6 \times 10^{-7}$ & $1.6 \times 10^{-5}$ \\
0.3 & $4.8 \times 10^{-7}$ & $5.7 \times 10^{-6}$ \\
0.4 & $3.2 \times 10^{-8}$ & $1.7 \times 10^{-5}$ \\
0.5 & $6.4 \times 10^{-7}$ & $5.2 \times 10^{-5}$ \\
0.6 & $9.1 \times 10^{-7}$ & $5.6 \times 10^{-6}$ \\
0.7 & $5.5 \times 10^{-7}$ & $2.3 \times 10^{-5}$ \\
0.8 & $3.2 \times 10^{-7}$ & $3.1 \times 10^{-5}$ \\
0.9 & $1.0 \times 10^{-6}$ & $3.0 \times 10^{-5}$ \\
1 & $9.7 \times 10^{-8}$ & $1.8 \times 10^{-5}$
\end{tabular}

Table 4 Computational results for Example. $2(\alpha=0.5)$

\begin{tabular}{lll}
\hline & $X_{n}=S_{0}^{6}, m=500$ & $X_{n}=S_{1}^{3}, m=500$ \\
\hline 0 & $3.0 \times 10^{-17}$ & $3.4 \times 10^{-17}$ \\
0.1 & $1.2 \times 10^{-7}$ & $7.6 \times 10^{-6}$ \\
0.2 & $3.1 \times 10^{-7}$ & $8.2 \times 10^{-6}$ \\
0.3 & $2.5 \times 10^{-7}$ & $1.6 \times 10^{-6}$ \\
0.4 & $3.8 \times 10^{-8}$ & $1.2 \times 10^{-5}$ \\
0.5 & $3.8 \times 10^{-7}$ & $3.2 \times 10^{-5}$ \\
0.6 & $5.3 \times 10^{-7}$ & $1.7 \times 10^{-5}$ \\
0.7 & $3.3 \times 10^{-7}$ & $2.6 \times 10^{-5}$ \\
0.8 & $5.6 \times 10^{-7}$ & $3.1 \times 10^{-5}$ \\
0.9 & $1.6 \times 10^{-7}$ & $3.1 \times 10^{-5}$ \\
1 & $2.5 \times 10^{-8}$ & $2.6 \times 10^{-5}$ \\
\hline
\end{tabular}

Numerical results of Tables 3 and 4 have a more accuracy with respect to Tables 1 and 2 . So, we prefer the numerical solution of singular integro-differential equation instead of Fredholm integral equation with logarithmic kernel.

\section{Conclusion}

In this article, we introduced a numerical method to combine kernel approximation with Galerkin method for solving integral equation with logarithmic kernel which it has a state of singularity. Also, we solved this problem by converting it to a simple form of singular integro-differential equation. we obtain a high accuracy to solve both of the above problems. 
Open Access This article is distributed under the terms of the Creative Commons Attribution 4.0 International License (http://creative commons.org/licenses/by/4.0/), which permits unrestricted use, distribution, and reproduction in any medium, provided you give appropriate credit to the original author(s) and the source, provide a link to the Creative Commons license, and indicate if changes were made.

\section{References}

1. Abdomen, E.M.A., Nasser, A.A.: On the numerical treatment of the singular integral equation of the second kind. Appl. Math. Comput. 146, 373-380 (2003)

2. Alpert, K.: A clases of bases in L2 for the sparse representation of integral operators. SIAM J. Math. 24, 246-262 (1993)

3. Aruchunan, E., Muthuvalu, M.S., Sulaiman, J., Koh, W.S., Akhir, M.K.M.: An iterative solution for second order linear Fredholm integro-differential equations. Malays. J. Math. Sci. 8, 158-170 (2014)

4. Balci, M.A., Sezer, M.: Hybrid Euler-Taylor matrix method for solving of generalized linear Fredholm integrator-differential equations. Appl. Math. Comput. 273, 33-41 (2016)

5. Chakrabarti, A., Berghe, V.G.: Approximate solution of singular integral equations. Appl. Math. Lett. 17, 553-559 (2004)

6. Kim, S.: Solving singular integral equations using Gaussian quadrature and overdetermined system. Appl. Math. Comput. 35, 63-71 (1998)

7. Ladopoulous, E.G.: Singular Integral Equations Linear and Nonlinear Theory and Its Applications in Science and Engineering. Springer, Berlin (2000)

8. Lifanov, I.K., Poltavskii, L.N., Vainikko, G.N.: Hypersingular Integral Equations and their Applications. Taylor \& Francis, London, New York (2004)

9. Martin, P.A., Rizzo, F.J.: On boundary integral equations for Crack Problems. Proc. R. A. 421, 341-345 (1989)

10. Noeiaghdam, S.: Numerical solution of $n$-th order Fredholm integro-differential equations by integral mean value theorem method. Int. J. Pure Appl. Math. 99, 277-287 (2015)
11. Pandey, P.K.: Non-standard finite difference method for numerical solution of second order linear Fredholm integro-differential equations. Int. J. Math. Model. Comput. 5, 259-266 (2015)

12. Prossdorf, S.: On approximate methods for the solution of onedimensional singular integral equations. Appl. Anal. 7, 259-270 (1977)

13. Rabbani, M.: Existence of solution and solving the integro-differential equations system by the multi-wavelet Petrov-Galerkin method. Int. J. Nonlinear Anal. Appl. 7(1), 207-218 (2016)

14. Rabbani, M., Maleknejad, K.: Using orthonormal wavelet basis in Petrov-Galerkin method for solving Fredholm integral equations of the second kind. Kybern. Int. J. Syst. Cybern. Manag. 41(3/4), 465-481 (2012)

15. Rabbani, M., Maleknejad, K.: A modification for solving Fredholm-Hammerstein integral equation by using wavelet basis. Kybern. Int. J. Syst. Cybern. Manag. 38(3/4), 615-620 (2009)

16. Shahsavaran, A., Shahsavaran, A., Fotros Paripour, F.: Numerical approach to solve singular integral equations using BPFs and Taylor series expansion. Int. J. Math. Model. Comput. 04(01), 77-91 (2014)

17. Shamooshaky, M.M., Assari, P., Adibi, H.: Wavelet method for the numerical solution of boundary integral equations with logarithmic singular kernels. Int. J. Math. Model. Comput. 04(04), 377-387 (2014)

18. Srivastav, R.P., Zhang, F.: Solution Cauchy singular integral equations by using general quadrature-collocation nodes. Appl. Math. Comput. 21, 59-71 (1991)

19. Sweilam, N.H., Khader, M.M., Kota, W.Y.: Numerical analytical. Probl. Eng. 2013, 1-7 (2013)

20. Wazwaz, A.M.: Linear and Nonlinear Integral Equations. Springer, Heidelberg (2011)

\section{Publisher's Note}

Springer Nature remains neutral with regard to jurisdictional claims in published maps and institutional affiliations. 